\title{
OBSERVATIONS UPON THE MOVEMENTS OF THE EYELIDS*
}

\author{
BY \\ G. GORDON \\ Laboratory of Physiology, Oxford University
}

THE human $M$. orbicularis oculi takes part in many patterns of movement. Some of these, like the corneal reflex, the narrowing of the palpebral orifice in bright light, or the adjustments which accompany upward or downward rotation of the eyeball, are connected in a comprehensible way with the eye itself. The muscle also plays its part in complex movements of the face, such as smiling. A third and particularly interesting type of movement is that of unconscious blinking, the pattern of which is undoubtedly related to visual perception (Hall, 1945), but which nevertheless occurs with about the normal frequency both in the blind, and in subjects in whom the cornea has been made anaesthetic (Ponder and Kennedy, 1928).

It is known from direct observation that there is some functional subdivision between the different anatomical regions of the orbicularis (Gad, 1883; Whitnall, 1921), and an attempt is made in this communication to amplify these earlier descriptions by means of electromyographic observations. It was found in the course of another investigation (Gordon and Holbourn, 1948) that the action potentials of single motor units could easily be recorded by means of wire electrodes placed upon the surface of the eyelids, and in this way one may get definite information about the activity of the motor neurones supplying this muscle. The opportunity has also been taken to investigate the activity of $M$. levator palpebrae superioris in the monkey.

\section{MethoDs}

HUMAN EXPERIMENTS.-The great majority of the observations were made upon my own eyelids. After some preliminary work, attention was limited to my left eye, of which a photograph was taken and a millimetre grid superimposed upon the photograph. The points from which observations had been made were recorded by reference to this grid.

The electrodes used for recording the action potentials of the orbicularis through the skin were described in an earlier paper (Gordon and Holbourn, 1948). Either one or

* Received for publication January 25, 1951. 
two pairs of these electrodes were used, and these were attached to holders kept in position by the hands. The electrodes only pick up large potentials when they rest on the thin skin which covers the lids and extends a short distance beyond them. In this region, it is often an advantage to push the points of the wires inwards, so that the skin is invaginated, and in this way one may explore the muscle almost as easily as with a subcutaneous needle electrode. Satisfactory records from the outer orbital regions could only be made with concentric electrodes; these were made from No. 17 dental needles, insulated up to the tip, and inserted through the skin.

The velocity of movement of the upper eyelid during blinking was recorded by an optical technique. A polished steel ball-bearing $(1.57 \mathrm{~mm}$. diam., weight $15 \mathrm{mg}$.) was stuck to the centre of the upper lid, $1 \mathrm{~mm}$. above its lower margin, and the skin of the lids and of the surrounding area was blackened with burnt cork. The ball-bearing was illuminated from above the eye with a 100-watt bulb, and a continuous record was made on moving photographic paper of the movements of the ball-bearing during blinking. The position of the ball with respect to the rest of the eye was checked by taking still photographs before starting the movement of the paper in the camera.

ANIMAL EXPERIMENTS.-A number of observations were made upon the levator and orbicularis of the baboon (Papio papio). The animal was anaesthetized with nitrous oxide, and then given sodium hexobarbitone intraperitoneally in a dose of $50 \mathrm{mg}$. per kg. body weight; the nitrous oxide was then stopped, and the anaesthetic maintained by further doses of $50 \mathrm{mg}$. hexobarbitone as required. The levator was exposed by carefully removing the roof of the orbit, starting from within the cranial cavity; the frontal pole of the cerebrum was undercut with bone forceps, but it remained undisturbed and the dura was not opened. A small concentric needle electrode, of a kind already described, was carefully inserted in the muscle. Other similar needles were inserted in various parts of the orbicularis, and simultaneous records were made from the two muscles.

Amplifiers.-Action potentials were amplified by resistance-capacity coupled pushpull amplifiers, and records made in a conventional way with cathode-ray tubes and a moving-paper camera. The published records have been re-photographed, but not re-touched. Measurements quoted were made from the original records, not from the reproductions.

\section{RESULTS}

(1) BLINKInG.-The word "blink" is used here to mean an extremely brief closing of the palpebral orifice, whether this particular intermittent type of movement is brought about voluntarily or involuntarily. There is some justification for describing these movements under one heading, because voluntary and involuntary blinks appear to involve the same parts of the eyelid musculature, and to have similar time-relations and electromyographic pattern. The movement involves both eyes; the upper lid moves briefly downwards and the lower lid upwards and nasally, both lids returning quickly to their resting positions.

The movement of my own upper eyelid has been studied in both voluntary and involuntary blinking by an optical recording method, the results being shown in Fig. 1 (opposite), and in the Table. 
TABLE

COMPARISON OF THE SPEED AND EXTENT OF MOVEMENT OF THE UPPER EYELID IN VOLUNTARY AND INVOLUNTARY BLINKING (G.G., LEFT EYE). SEE ALSO FIG. 1.

\begin{tabular}{c|c|c|c|c|c}
$\begin{array}{c}\text { Type of } \\
\text { Blink }\end{array}$ & $\begin{array}{c}\text { No. of } \\
\text { Observations }\end{array}$ & \multicolumn{2}{|c|}{ Extent of Movement } & \multicolumn{2}{|c}{ Time to Crest of Curve } \\
\hline \begin{tabular}{c|c|c|c|c} 
Mean \\
(mm.)
\end{tabular} & $\begin{array}{c}\text { Standard } \\
\text { Error }\end{array}$ & $\begin{array}{c}\text { Mean } \\
\text { (msec.) }\end{array}$ & $\begin{array}{c}\text { Standard } \\
\text { Error }\end{array}$ \\
\hline Voluntary $\ldots$ & 23 & 10.45 & \pm 1.04 & 50.26 & \pm 8.81 \\
Involuntary & 8 & 11.04 & \pm 0.25 & 52.94 & \pm 13.52 \\
\hline
\end{tabular}

A statistical analysis of the mean figures quoted in the Table showed no significant difference between the two types of blink. The values of standard error indicate that variations in the pattern of movement exist within each group; the curves in Fig. 1 have been selected to show the approximate range of this variation.

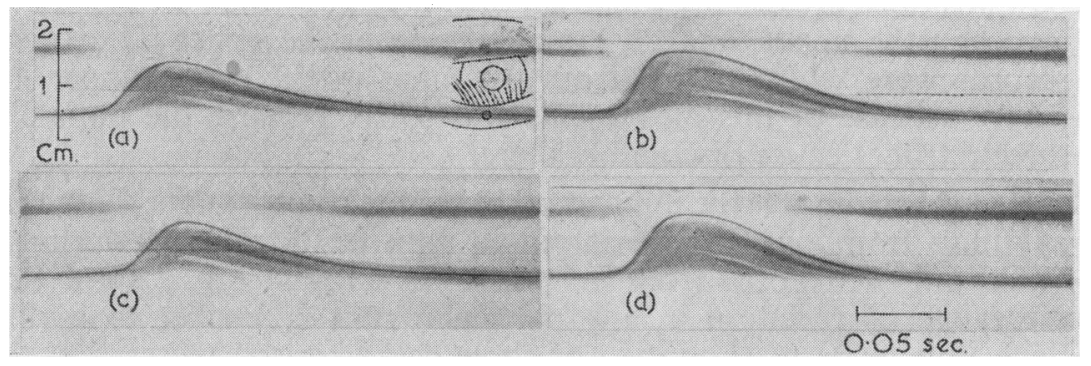

FIG. 1.-Blinking. Records of the movement of an illuminated ballbearing attached to the upper lid. (a) and (b) involuntary blinks ; (c) and $(d)$ voluntary blinks. The records have been inverted for reproduction, an upward movement in the figure representing a downward movement of the lid. The inverted diagram of the eye in $(a)$ was traced from a still photograph, and shows accurately the position of the ball-bearing in relation to the rest of the eye. The head had been rotated through $10^{\circ}$ in the coronal plane, raising the left eye from the horizontal, so as to record a true vertical movement of the upper lid.

The onset of the movement is not abrupt; in many records there is a slight inflection of the curve after the lid has moved down about $1 \mathrm{~mm}$., and thereafter the movement is rapid. The significance of this inflection will be considered later in this paper. After the crest, the curve of relaxation is smooth, and the point of completion of the movement is not easy to determine accurately. In four recorded blinks, two voluntary and two involuntary, a sufficient stretch of level baseline was present to allow approximate determinations of the total duration of the blinks: the mean figure was 288 msec. ( \pm 7 per cent.). It was also observed that under similar 
conditions to those of the experiment, my left upper eyelid can descend about $2 \mathrm{~mm}$. without interference with vision, and from the same four records as those mentioned above, the period during which vision is interfered with by the blinks was estimated as $130 \mathrm{msec}$. ( \pm 3.8 per cent.). This figure is of the same order as that given by Lawson (1948) as the value for total period of blackout of his own pupil during blinking $(0.1 \mathrm{sec}$.$) .$

Muscle action potentials associated with blinking can be detected from the whole surface of both upper and lower eyelids, and over the extensions of the palpebral muscle surrounding the inner and outer canthi. The potentials appear as bursts of activity, lasting for periods up to about $55 \mathrm{msec}$., and usually consisting of high-frequency. trains of impulses in a number of motor units, firing asynchronously, but records can be made from single isolated units after the position of the electrodes has been suitably adjusted. If the electrodes rest on a part of an eyelid where considerable movement is taking place, the movement causes an artefact in the potential record, so that the best positions are usually found on the lower lid, which moves much less than the upper, and on the nasal side of the upper lid. Good records were seldom made from the main extent of the upper lid, partly because of this movement, and partly because the points of the electrodes cause discomfort there.

Records from a large number of individual motor units show that anything from one to five impulses may be discharged during a blink, but the records from four different units in Fig. 2 show that the range of variation in any particular unit is smaller than this. Each unit tends to show two or three characteristic patterns in the temporal grouping of the impulses, and in a large number of records from any particular unit, each pattern recurs a number of times in almost identical form; the records in Fig. 2 have been selected to show the range of temporal pattern which was found in each of the four units. One particular type of grouping which calls for comment is seen in Fig. 2, $a$ and $c$; the first two impulses are very close together, and the third occurs after a considerably longer interval. The occurrence of this interval suggests that the first pair of impulses may result from a "double discharge" of the motor neurone, an effect which is found in human limb muscles (Gilson and Mills, 1941; Denslow, 1948), and which has an initial accelerating effect upon a contraction (Gordon and Holbourn, 1949). The tendency for this type of grouping to occur introduces some difficulties in the measurement of the frequencies at which these motor units discharge during blinking, but it was decided arbitrarily to estimate frequencies only where three or more impulses were fired, whether or not the suggestion of a double discharge and compensatory interval was present. The frequency was then estimated over the first three 


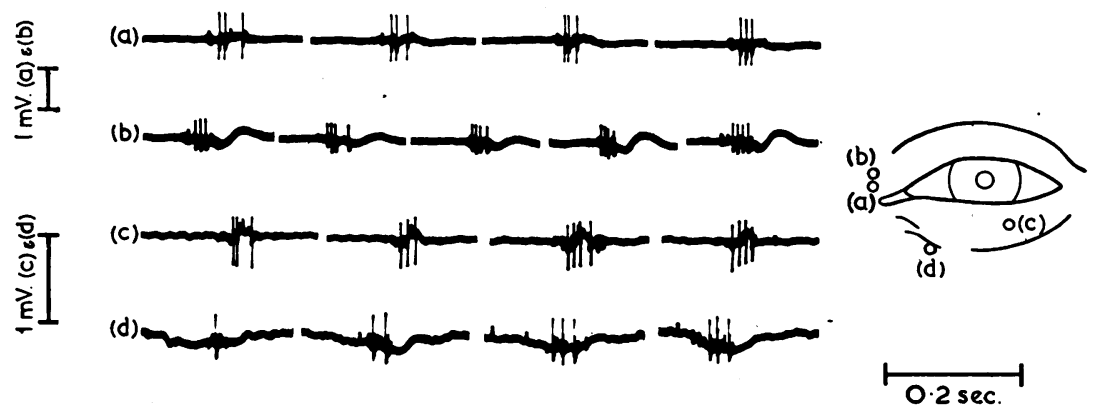

FIG. 2.-Blinking. Records of the action potentials of four different motor units, $a, b, c$, and $d$, during voluntary blinking, several examples being shown from each unit. The anatomical positions from which these records were made are shown in a diagram.

Frequencies of firing measured over first three impulses :

(a) $\left|59^{*}\right| 8^{*}\left|118^{*}\right| 125|-|$ impulses per sec. Total observed range 59*-125 per sec.

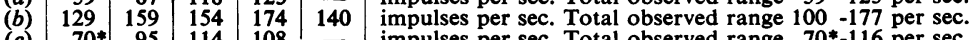

(c) 70* 95 114 108 - impulses per sec. Total observed range $70^{*}-116$ per sec.

impulses per sec. Total observed range $59-105$ per sec.

*iThese observations suggested the presence of fa double discharge and compensatory interval (see text).

impulses of the burst of activity. With these criteria, the range of frequencies over a large number of motor units lay between 45 and 182 impulses per sec., the range for any particular unit being considerably narrower than this (see Fig. 2, legend).

The motor units lying nearest to the margins of the eyelids generally discharge at the highest frequencies, but it is difficult to obtain satisfactory records from this region, since the number of units taking part in blinking is very great there, and the records are often complex. For this reason, it is possible that higher frequencies of discharge occur in these marginal units than has been recorded in this paper.

The majority of the motor units which respond in blinking have shown no evidence of taking part in other types of contractionthat is to say, attempts to make them contract by sustained voluntary effort are not successful, and no voluntary grading of the blink contraction seems possible. Units of this type appeared to take no part in attempts to close only one eye (winking). Some units in the outer regions of the lids, however, and a few even in the superior orbital part of the muscle, can respond both in blinking, in unilateral winking, and in graded voluntary contraction; these units show relatively low impulse-frequencies during blinking (e.g., Fig. 2,d), and their threshold in tonic voluntary contraction is high in relation to other units responding during tonic voluntary effort (see the following section of this paper). These observations, therefore, suggest an exclusiveness of the " blink" units nearest to the margins of the lids, although it cannot be said categorically that they take no part in maximal tonic contractions when the whole eye is strongly 
screwed up, since continuous observation of any particular unit under these circumstances is impossible; certainly their threshold to tonic voluntary activation is, at least, very high.

In this type of experiment, the anatomical position of a motor unit can only be assessed by moving the electrodes until the potentials of the unit reach maximal amplitude. With this criterion, it must be assumed that the great majority of the units responding in blinking belong to the palpebral part of the orbicularis, and are most numerous in its inner portions-those nearest to the margins of the lids.

(2) Sustained Voluntary Contractions.-A variety of movements of the eyelids can be brought about by sustained voluntary effort, ranging from a minimal narrowing of the lid aperture to a maximal screwing-up of the whole eye. These movements may be unilateral or bilateral.

In slight narrowing of the aperture, the lower lid moves upwards and a little nasally, but without the marked nasal direction which is seen in blinking. The contraction appears to begin in the lower regions of the lid, but when the effort increases, regions below the lid also contract. The upper lid moves downwards, but the contracting muscle is not seen at first, since the upper part of the lid is out of sight. The motor units of lowest threshold in these slight sustained contractions appear, from action potential studies, to lie in the regions of the upper and lower eyelids furthest from the lid margins. Their discharges can be graded, by voluntary effort, from single isolated impulses to rhythmic discharges, with an upper limit of frequency, in strong contraction, of about 50 per sec. Their properties resemble those of the motor units in limb muscles under similar volitional drive. No activity has been seen in these lowthreshold units during blinking, and when a blink is superimposed upon a slight tonic contraction in which one of these units is involved, the tonically contracting unit rarely shows any increase in frequency of firing during the occurrence of the blink; sometimes a drop in frequency occurs immediately after the blink has taken place (Fig. 3, $b, c$, and $d$ ).

More units of this low-threshold type are recruited into activity as the contraction proceeds; when the effort becomes still stronger, discharges occur in a group of motor units which respond both in blinking and in sustained contractions. The activity of a unit of this type is shown in Fig. 2, $d$, which was referred to in the previous section. A similar unit is shown in Fig. 3, $a$, where it becomes active half-way through the record with an increase in voluntary effort, and the same unit fires in the blink responses of Fig. 3, b, and $c$. This particular unit was easily identified in different responses by the polyphasic configuration of its action potentials, an example of which is shown on an expanded time-scale in Fig. $3, b^{\prime}$. 
(a)

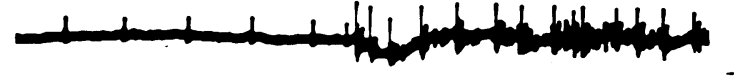

(b)

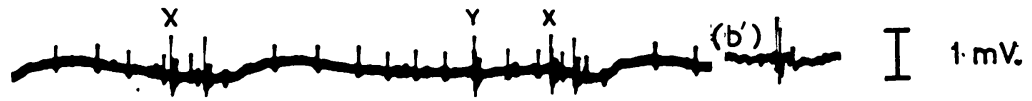

(c)
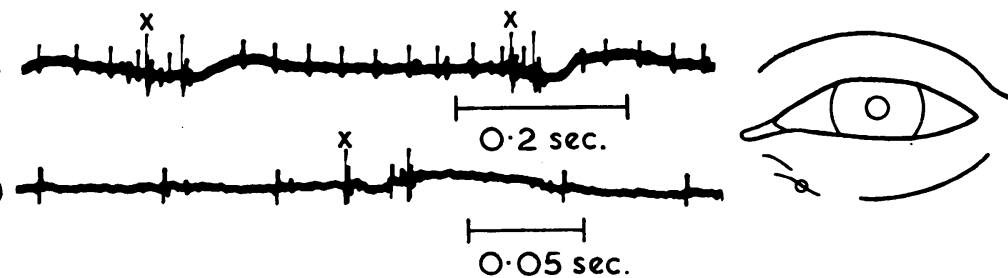

FIG. 3.-Sustained voluntary activity. Records of the action potentials of two motor units, made from the position shown in the diagram. The first unit with small potentials fires during slight sustained effort, but takes no part in blinking; the second unit with large potentials fires during strong sustained effort, and also during blinking.

(a) slight sustained effort, with a sudden increase in effort half-way through the record.

(b) and $(c)$ moderate sustained effort, showing at one point $(y)$ a single discharge of the second unit. The points marked $x$ represent blinks, in which the second unit is active.

(d) record made at higher speed, after slight adjustment of the electrode position.

$\left(b^{\prime}\right)$ refers to records $(a)$ to $(c)$, and shows a record of one action poten-

tial of the second unit made at the same speed as record $(d)$; it is useful in identification.

In strong contractions, in which the eye begins to be screwed up, activity is found in the outer (orbital) regions of the orbicularis. In a series of observations which were made with concentric needle electrodes, placed in this muscle about $8 \mathrm{~mm}$. below the inferior orbital margin, no activity whatever was found during blinking. A single motor unit which was identified in this region took part in a variety of voluntary movements, responding during elevation of the angle of the mouth at low volitional threshold, during screwing up of the eye at a higher threshold, and during dilatation of the nostril at still higher threshold; it also responded readily in smiling.

The superior orbital region of the orbicularis was also explored with a subcutaneous electrode, inserted $10 \mathrm{~mm}$. above the superior orbital margin to a depth of about $5 \mathrm{~mm}$. The great majority of the units encountered here responded only during screwing up of the whole eye and during smiling or frowning. One unit was of a type referred to in para. 3 of this section, responding at low frequency in blinking, as well as during moderate sustained voluntary effort. It also responded tonically when the gaze was directed downwards as far as possible.

(3) Activity Related to Change of Ocular Fixation.-When the gaze is directed upwards, the upper and lower lids rise, following closely the movement of the iris. The converse effect occurs on 
looking downwards. When the eyeballs rotate quickly, the initial adjustment in the position of the eyelids is accompanied by brief motor unit discharges in each lid, the units involved belonging to the group described in Section 1 (Fig. 4, $a$ to $c$ ). This may involve an actual blink, which often accompanies changes of ocular fixation (Hall, 1945). The number of units firing is often small, however, and the movement may not be large enough to close the lids. If the eyeballs rotate slowly, this brief initial discharge may not occur (cf. Fig. 4, $c$ and $d$ ).

This initial burst of activity is followed, when the gaze is upwards, by sustained activity in a small number of motor units in the lower part of the lower lid, which only cease to fire when the eyes look down again. These units appear to take no part in blinking, but belong to the group described in Section 2, which have a low threshold in slight sustained voluntary effort. The frequency of discharge in these units increases with increased upward rotation of the eyeball, and at the same time more units are recruited into activity.

No sustained motor unit discharges have been detected with surface electrodes on the upper lid during the converse process of looking downwards. One unit has been detected with an inserted electrode in the superior orbital region of the orbicularis, which responded only on extreme downward direction of the gaze. A considerable part of the downward movement of the upper lid in these circumstances is presumably brought about by partial relaxation of the levator.

(4) CoRneal Reflex.-A iew observations have been made on corneal reflexes induced by lightly touching the cornea with wisps of cotton wool. Two types of motor discharge have been observed. The first occurs in the group of motor units which also discharge in blinking, and the pattern of activity in the reflex is very similar to that of blinking, being bilateral, and consisting of brief high-frequency bursts of impulses. The stimulus was not quantitatively controlled, but a number of observations gave the impression that an increase in the stimulus caused more units to fire, but that the characteristics of the discharge of any single unit were little affected. Persistence of the stimulus, however, when the cotton wool was not immediately removed from the cornea, often caused one or more repetitions of the reflex discharge.

The second type of activity consisted of a discharge of greater duration and lower frequency in a few units of the type which have a low threshold in sustained voluntary activity, but which take no part in blinking. This is most conspicuous in the peripheral portion of the lower lid, and may occur unilaterally.

The first and second types of discharge may occur together in a 
(a)
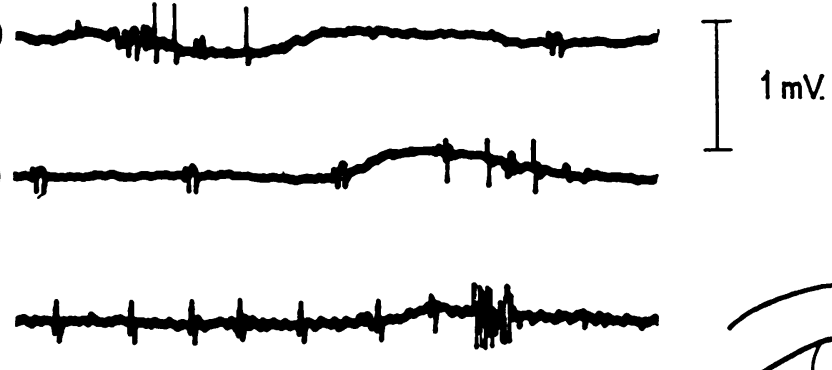

(b)

(c)

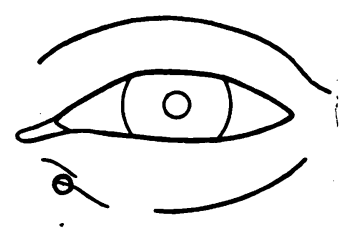

(d)

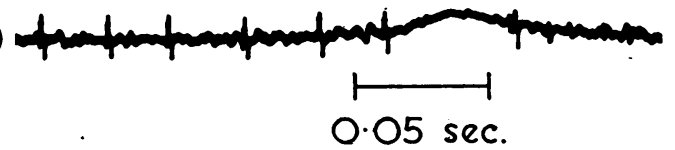

Fig. 4.-Changes of fixation. Lower lid.

(a) and $(b)$ show two parts of a record in which the gaze was directed quickly upwards (at beginning of $(a)$ ), and then quickly downwards (at end of $(b))$. The small polyphasic potentials are those of a 'tonic' unit, the large potentials those of a 'blink' unit.

Records $(c)$ and $(d)$ were made from a new electrode position near to the first. (c) shows a quick downward direction of the gaze, in which a blink occurred; $(d)$ shows a slower downward direction, without a blink. In each case the gaze had been directed upwards for several seconds previously.

reflex if the stimulus is strong: weak stimuli sometimes induce discharges of the first type only.

(5) ANIMAL EXPERIMENTS.-The experimental results obtained from the human eyelids are precise enough to allow a tentative classification of the various parts of $M$. orbicularis oculi according to the types of movement in which they are involved. The movements which occur spontaneously, or which may be elicited, in the anaesthetized animal are of such a limited kind that it seemed that little would be gained by attempts to relate them to the movements in the conscious human being. The only matter upon which no evidence could be obtained in the human being was the part which is played by $M$. - levator palpebrae superioris, particularly during blinking: it was not known, for instance, whether the levator remains active during a blink, or is partially or completely relaxed.

An experiment was carried out upon a baboon (Papio papio), anaesthetized with hexobarbitone. These animals, when conscious, blink spontaneously in a way closely resembling that of the human being, and, unlike most of the smaller monkeys, their eyes may remain open, and some blinking may continue, when the animal is anaesthetized. In very profound barbiturate anaesthesia, especially under "Dial", the eyes remain closed. The levator was exposed and electrodes inserted in it (see METHODs): it was found that 
while the eyes remained open, the activity of single motor units could be detected in the muscle, discharging at frequencies between 15 and 50 impulses per sec. The beginning of each spontaneous blinking movement was accompanied by complete cessation of activity in the levator, which remained inactive for 240 to $560 \mathrm{msec}$. (Fig. 5); this indicates a reciprocal relationship between the levator and the orbicularis, even in this extremely brief movement. It was also found that in seven out of fourteen successive blinks there was a drop in the frequency of firing of a single unit in the levator which could be detected some 35 to $85 \mathrm{msec}$. before total relaxation of the muscle occurred (Fig. 5, $a$ and $c$ ). This partial relaxation of the levator will be referred to below in relation to the slow initial movement of the upper eyelid in the human blink.

In very profound anaesthesia, the eye was closed, and no activity could be detected either in the levator or the orbicularis. When the lids were slightly separated, and jets of air blown on to the cornea, reflex electrical activity could sometimes be detected in the orbicularis, even though no movement could be seen. This consisted of an immediate brief discharge at high frequency, followed by tonic activity which was sustained for a variable period. Observations made during repeated movements of the needle electrode suggested strongly that the initial responses were of greatest amplitude in the portion of the muscle nearest to the margins of the lids, and the sustained responses in more peripheral portions. Unfortunately, however, the needle electrode did not allow such precise localization as the surface electrodes which were used upon the human eyelid, and no clear-cut anatomical distinction could be made.

\section{Discussion}

The great variety of the movements in which the eyelids are concerned has been discussed in detail by Gowers (1879), and by Whitnall (1921), and it is proposed only to deal here with the types of activity described above. The results support, in a broad sense, the conclusion of Gad (1883) that, within the eyelids, the innermost (pretarsal or epitarsal) fibres are concerned with the movement of blinking, while the more peripheral (preseptal or peritarsal) fibres are concerned with sustained narrowing of the palpebral orifice. There seem, however, to be three main functional groups of motor units, and although the general arrangement is that described by Gad, no group is entirely confined to one anatomical portion of the muscle. The first group contains those units which seem to be exclusively concerned in blinking or in the initial movement of the corneal reflex: it is confined to the eyelids, and the greatest number of units lie in the innermost (pretarsal) portions. There is no evidence that the units of this group can take part in unilateral 


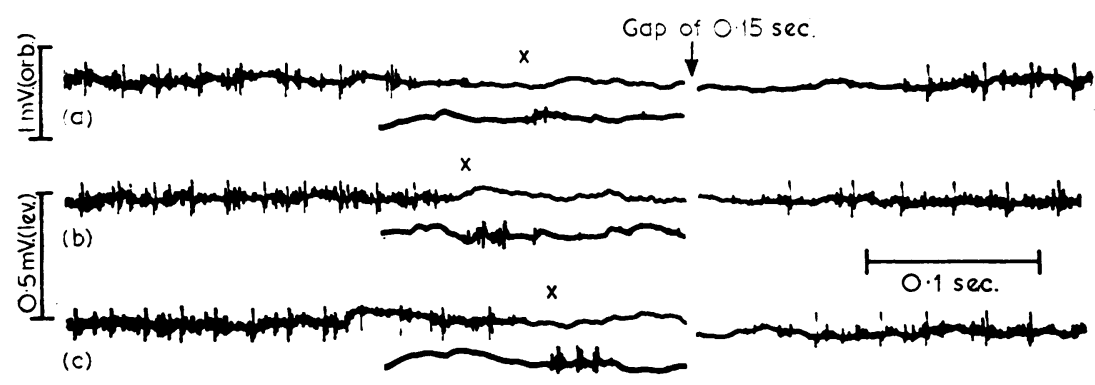

Fig. 5.-Spontaneous blinking in the baboon. Records from the levator (upper continuous line) and the orbicularis (lower insert) during three blinks $(a)$ to $(c)$. The onset of each blink is marked $x$. At the point marked by the vertical arrow, a piece of the record corresponding to $0.15 \mathrm{sec}$., during which no activity was observed in the levator, has been omitted in order to save space.

The intervals between cessation of activity in the levator and the onset of activity in the The intervals between cessation of activity in the levator and the onset of activity in the
orbicularis depend upon the latencies of the particular units selected for recording. In another series of records with different electrode positions no appreciable interval was seen.

activity. The units of the second group are concerned both with blinking and with the various kinds of sustained activity which have been described, their threshold in sustained activity being fairly high; these are found in the peripheral (preseptal) regions of both eyelids, and, in smaller numbers, in the superior orbital part of the muscle. The third group contains units which respond only in sustained activity; those of the lowest threshold lie in the preseptal regions of both lids, those of higher threshold in the orbital parts of the muscle. The units of the second and third groups can act unilaterally.

In the movements of the eyelids which accompany upward or downward rotation of the eyeball, a small number of units of the second and third groups, just described, play a part: the muscular activity is very small, and Gowers (1879) believed that no such activity occurred. It seems likely that, as Gowers suggested, the movement of the upper lid in such circumstances is mainly due to changes in the level of tonic activity in the levator.

The movement of blinking calls for particular comment. The great majority of the motor units concerned discharge only brief volleys of impulses and at very high frequencies. The maximal frequency of discharge in the human muscles which have previously been examined during voluntary contraction is believed to be about 50 per sec., whereas frequencies up to 182 per sec. have been observed in the orbicularis during blinking; this is of the order of the highest frequencies of discharge observed by Reid (1949) in the cat's extra-ocular motor neurones, under reflex drive. The extraocular muscles have a very brief contraction time (Cooper and Eccles, 1930), and this may also be true of the pale muscle-fibres 
making up the pretarsal part of the orbicularis. This extremely rapid movement is capable of intermittence at very high frequencies, Walter (1941) having recorded voluntary blinking in man at a frequency of 390 per minute: the movement of this part of the muscle is also peculiar in that although it can be initiated voluntarily, it cannot subsequently be modified voluntarily or sustained. The maintenance of the narrowed or closed position after a blink depends on the activity of units in other parts of the muscle and upon relaxation of the levator: if a conscious effort is then made to relax, a state of rest may be reached in which the eyes are lightly closed and no activity can be detected in the orbicularis, a state which presumably occurs during sleep.

If it is accepted that the pathway for the corneal reflex is subcortical, then the act of blinking is likely to owe its inherent quality of intermittence to a subcortical mechanism also, since the discharges of motor neurones in blinking are similar to those of the same neurones in a corneal reflex. Nothing is known of the method by which such a subcortical mechanism is " triggered " in voluntary or involuntary blinking, but some of the results described above suggest that the first peripheral change to occur in a blink may be a partial relaxation of the levator rather than a contraction of the pretarsal part of the orbicularis. This occurred in about half the records made from the levator of a baboon (Fig. 5), and it is possible that a partial relaxation of the levator may explain the small and slow initial movement which occurs in optical records of the human upper lid during blinking (Fig. 1). The preliminary partial relaxation of an antagonist is not a recognized feature of muscular contractions, but it may have significance in this unusual movement in that it allows the orbicularis to contract from the start against a reduced resistance, and this would shorten the time during which vision is disturbed.

\section{SUMMARY}

(1) An electromyographic study has been made of the activity of the human $M$. orbicularis oculi. Three main functional groups of motor unit have been identified - those responding in blinking and in the corneal reflex, those responding both in blinking and in sustained activity, and those responding only in sustained activity.

(2) There is considerable anatomical overlap between the distributions of these three groups of motor unit. Those of the first group lie mainly in the pretarsal regions, and those of the second group mainly in the preseptal regions, while those of the third group extend from the preseptal to the orbital regions.

(3) Particular attention has been given to blinking, and optical records have been made of this movement. In blinking, the units 
of the first group discharge brief bursts of impulses at very high impulse-frequency (up to 182 per sec. in a single unit): they seem to take part in bilateral movement only. Voluntary initiation of these discharges is possible, but they cannot be voluntarily graded or sustained.

(4) The units of lowest threshold in the second and third groups (mainly preseptal) take part in voluntary or involuntary narrowing of the lids, in the slow component of the corneal reflex, and in tonic contractions of the lids in relation to vertical changes in ocular fixation. Units of higher voluntary threshold (mainly orbital) take part in screwing up the eye: some of these latter units have a low threshold in complex movements like smiling.

(5) The activity of $M$. levator palpebrae superioris has been studied in the anaesthetized baboon. Motor units in this muscle discharged at frequencies between 15 and 50 impulses per sec. when the eyes were open, this discharge being promptly inhibited when a blink occurred. In about half the records, a drop in frequency occurred 35-85 msec. before the blink; this is discussed.

I am very grateful to Dr. J. A. V. Bates for introducing me to an optical technique for recording eyelid movement similar to the one which I have used. I would also like to thank Mr. P. Philbin for his technical assistance.

\section{REFERENCES}

COOPER, S., and ECCLES, J. C. (1930). J. Physiol., Lond., 69, 377.

DensLow, J. S. (1948). J. Neurophysiol., 11, 209.

GaD, J. (1883). Arch. Anat. Physiol. Lpz. Physiol. Abt., Suppl.: “ Festschrift für du Bois-Reymond ", p. 69.

Gilson, A. S., and MilLs, W. B. (1941). Amer. J. Physiol., 133, 658.

Gordon, G., and Holbourn, A. H. S. (1948). J. Physiol., Lond., 107, 456.

-,

Gowers, W. R. (1879). Med.-chir. Trans., 62, 429.

HALL, A. (1945). British Journal of Ophthalmology, 29, 445.

LAWSON, R. W. (1948). Nature, Lond., 161, 154.

Ponder, E., and Kennedy, W. P. (1927). Quart. J. exp. Physiol., 18, 89.

REID, G. (1949). J. Physiol., Lond., 110, 217.

WALTER, W. G. (1941). Arch. néerl. Physiol., 25, 601.

WhItNALL, S. E. (1921). "The Anatomy of the Human Orbit". Oxford University Press, London. 